\title{
Medición de calidad de servicio mediante el modelo SERVQUAL: el caso del Juzgado de Garantía de la ciudad de Puerto Montt - Chile
}

\author{
Service quality measurement: the case of the Guarantee \\ Court from the city of Puerto Montt - Chile \\ Francisco Ganga Contreras $^{1} \quad$ Nancy Alarcón Henríquez ${ }^{2} \quad$ Liliana Pedraja Rejas ${ }^{3 *}$ \\ Recibido 11 de noviembre de 2018, Aceptado 24 de junio de 2019 \\ Received: November 11, 2018 Accepted: June 24, 2019
}

\begin{abstract}
RESUMEN
Este artículo considera la creciente relevancia que ha tomado el tema de la calidad como un imperativo estratégico para asegurar la viabilidad y el éxito organizacional, por esto su propósito central es presentar los resultados de la calidad del servicio medido en el Juzgado de Garantía de la ciudad de Puerto Montt a través del modelo SERVQUAL. Para tal efecto, se construyó un cuestionario que recogió 16 indicadores que representan las cinco dimensiones señaladas por Parasuraman, Zeithaml y Berry, medidos en una escala Likert de 5 puntos, el cual fue aplicado a personas mayores de 18 años que se encontraban como usuarios del Juzgado de Garantía en las fechas de diciembre del 2013 y junio del 2018. Los niveles de expectativas más elevados se encontraron en las dimensiones: confiabilidad, seguridad y aspectos tangibles, mientras que aquellas percibidas como de mejor calidad fueron empatía y aspectos tangibles. En general, los niveles de calidad del servicio se mostraron desfavorables o negativos debido principalmente a que no fueron superadas las expectativas del usuario por sus percepciones. Esta información es valiosa ya que puede ser utilizada por la organización para mejorar aquellos atributos de mayor relevancia para los usuarios.
\end{abstract}

Palabras clave: Calidad de servicio, SERVQUAL, expectativas, percepciones.

\begin{abstract}
This article starts with the growing relevance that the topic of quality has taken as a strategic imperative to ensure the viability and organizational success. The main purpose is to present the results of the perceived service quality measured in the Guarantee Court of the city of Puerto Montt through the SERVQUAL model. To this end, a questionnaire was collected with 16 indicators, representing the five dimensions indicated by Parasuraman, Zeithaml and Berry, measured on a Likert scale of 5 points, which was applied to people over 18 who were users of the Court of Guarantee on the dates of December 2013 and June 2018. The highest levels of expectations were found in the dimensions: reliability, security, and tangible aspects, while those perceived as having the highest quality were empathy and tangible aspects. In general, service quality levels were unfavorable or negative, mainly because the user's expectations for their perceptions were not exceeded. This information is valuable because it can be used by the organization to improve those attributes of greater relevance to users.
\end{abstract}

Keywords: Service quality, SERVQUAL, expectations, perceptions.

\footnotetext{
1 Universidad de Los Lagos. Departamento de Ciencias del Desarrollo. Santiago, Chile. E-mail: fganga@ulagos.cl

2 Universidad de Los lagos. Departamento de Gobierno y Empresa. Puerto Montt, Chile. E-mail: n.alarcon@ulagos.cl

3 Universidad de Tarapacá. Departamento de Ingeniería Industrial y de Sistemas. Arica, Chile. E-mail: 1pedraja@uta.cl

* Autor de correspondencia: lpedraja@uta.cl
} 


\section{INTRODUCCIÓN}

La gestión de calidad en las instituciones del Estado, se refiere sobre el eficiente y buen servicio que puedan brindar las diversas organizaciones públicas hacia una sociedad cada día más exigente, con mayores problemas a resolver y con necesidades en distintas áreas tales como: social, política, económica, cultural, de seguridad, etc. Ante ello, el Estado debe responder y, como es sabido, con recursos que no son ilimitados. Se entiende que para ello se deben mantener instituciones sólidas, legítimas y eficientes generando un adecuado desarrollo institucional que asegure el bien común hacia una nación. En este sentido, se requiere que las organizaciones del Estado se vayan adaptando a los cambios que presente y requiera la sociedad del conocimiento para el beneficio de la paz y la estabilidad.

En el mundo actual, los cambios sociales han forzado a las instituciones a establecer "compromisos éticos" que les permitan compararse con otro tipo de organizaciones en cuanto a prestigio. Dicho objetivo se podrá alcanzar siempre y cuando se plantee la "calidad" como una táctica maestra para lograr un funcionamiento óptimo [1]. Teniendo en cuenta que la entrega de un servicio de calidad es la clave del éxito en las industrias de servicios, ya que, en estas, la mejora de la calidad es el factor principal que afecta la satisfacción del consumidor [2].

La calidad de servicio ha sido central para el Poder Judicial chileno durante los últimos años, realizándose permanentes esfuerzos para mejorar la excelencia de la atención recibida por los usuarios en los distintos tribunales del país. En efecto, a modo de ejemplo, en su plan estratégico 2011-2015 dan cuenta de ello, indicando la importancia de mejorar su servicio constantemente a través de nuevas prestaciones para los usuarios y de la construcción de un modelo de atención de usuarios, el cual permite enunciar, en forma preliminar, los atributos del servicio a entregar.

De acuerdo a la Real Academia de la Lengua Española, la calidad se define como: "propiedad o conjunto de propiedades inherente a algo, que permiten juzgar su valor" [3]. Trasladado al ámbito de los servicios prestados por el Estado, la calidad se asocia a la entrega de un conjunto de prestaciones como los demanda el ciudadano, adaptándose a sus exigencias. El concepto de calidad transmite la idea de compromiso con este, que se traduce en hacer efectivo un contrato social por el que las administraciones públicas buscan activamente adaptarse a las necesidades de los ciudadanos que son su razón de ser.

La gestión centrada en la calidad total debe contener actividades de mejora continua que involucren a todos los procesos de una organización, en un esfuerzo en la dirección de mejorar el desempeño de cara a los desafíos del mercado [4]. Hoy en día, cualquier responsable de la gestión pública o privada debe reconocer la importancia que tiene la percepción de los clientes sobre sus productos. Dicho de otro modo, más allá de los logros productivos (medidos mediante los indicadores objetivos) la percepción que tienen los usuarios y la población general señala el lugar que ocupa ese servicio público en la escala de estima que tiene esa sociedad [5].

El sistema judicial constituye un medio para conseguir el cumplimiento de una función primordial del Estado (la protección de los derechos de los ciudadanos mediante la resolución de los conflictos entre particulares y entre estos y los poderes públicos). La prestación de dicha función debe realizarse siempre en el cumplimiento del fin de la justicia. Desde esta perspectiva, el ciudadano (usuario del sistema judicial) se convierte en el protagonista real de dicho sistema, pues es el sujeto beneficiario del mismo y cuyo goce le pertenece, desplazando de dicho protagonismo, al juez y al resto de los profesionales que asisten a su administración.

Por consiguiente, las encuestas a los usuarios son un instrumento de carácter insustituible en la planificación de los esfuerzos en las políticas públicas. No obstante, cuando se habla de calidad en los tribunales de justicia, la percepción y valoración por parte de los usuarios tiene un peso aún mayor al que alcanzan en otros servicios públicos, como la salud o la seguridad social [5]. El grado de confianza que los tribunales hayan logrado de los usuarios (ciudadanos que han sido involucrados en su actividad ya sea como imputados, víctimas, testigos, demandantes o demandados) es una magnitud de primer orden para legitimar la actividad judicial, esto es, ser una instancia legítima para la resolución de conflictos.

En esta lógica, el presente estudio tiene como objetivo principal identificar los resultados de 
medición de la calidad de servicio realizado en dos momentos del tiempo en el juzgado de garantía de la ciudad de Puerto Montt - Chile, basada en la percepción del usuario, que permita conocer sus requerimientos, medir su nivel de satisfacción y proponer las acciones necesarias para aumentar dicho nivel. Para ello, se utilizó un instrumento basado en el modelo SERVQUAL, con 16 indicadores con las cinco dimensiones señaladas por Parasuraman, Zeithaml y Berry [6] medidos en una escala Likert de 5 puntos.

\section{MARCO TEÓRICO}

La calidad es un término que todos los consumidores creen entender pero que difícilmente pueden conceptualizar. En definitiva, y de acuerdo a lo planteado por diversos autores, la calidad es un constructo que admite múltiples significados [710], debido a que es una creación conceptual para explicar ciertos fenómenos abstractos de forma lógica y coherente [11]. Dentro de este contexto, la calidad es un término relativo multidimensional que supone acepciones diferentes, tanto en el tiempo como en función de quien lo utilice, pues implica el necesario juicio de valor individual y colectivo [12].

Según Vera y Trujillo [13] para poder mejorar la calidad en un servicio resulta esencial tener claro qué es y cómo medirla. En este sentido, referirse al enfoque de calidad dice relación con hacer mención de los diversos factores que influyen en la relación usuario y organización, frente a los bienes y servicios que estos entregan a los usuarios. Allí confluye un contexto complejo que refleja las expectativas que esperan y deben ser cumplidas íntegramente por quienes componen la organización.

Es así como en el sector de la educación, la calidad del servicio es un tema central toda vez que las instituciones deben llevar a cabo sus proyectos en un enfoque de aseguramiento de esta [14]. Es por esto que Pedraja y Rodríguez [15] señalan que, en las instituciones educativas, el garantizar la calidad ha pasado a ser un imperativo para poder competir en el mercado, donde la percepción de la misma depende directamente del stakeholder que la evalúa. De esta forma, en los últimos 25 años han emergido distintos procesos para el aseguramiento de la calidad en la educación [16].
En todo caso, el interés actual en diversos sectores industriales por la calidad de los servicios obedece a diversas razones basadas en el concepto de excelencia, como son a) aumentar la incorporación de nuevos clientes, b) fidelizar a los usuarios/clientes actuales y potenciales, c) ofrecer oportunidades objetivas para la mejora y el desarrollo organizacional/institucional y d) optimizar la relación costes/beneficios así como la imagen institucional, fortaleciendo la permanencia de sus miembros [17]. Si las empresas conocen lo que necesitan y desean los clientes, se logrará generar valor y con ello se crearán productos y servicios de calidad.

La calidad de los servicios es más difícil de evaluar que la calidad de los bienes, ya que la ausencia de evidencia o elementos tangibles obliga a los usuarios a depender de otros elementos. Es por esto, que poseer un conocimiento profundo sobre las expectativas del cliente es vital para los proveedores de servicios, debido a que saber lo que espera el usuario es el primer paso y, posiblemente, el más crítico para brindar un servicio de buena calidad [18]. La evaluación de calidad del servicio no está hecha solamente de los resultados del mismo, sino que también depende de la evaluación del proceso de entrega. Más aún, el cliente puede llegar a dar un mayor énfasis a la interacción con el trabajador con quien se contacta directamente más que al servicio entregado en sí [19].

Las características diferenciadoras de los servicios implican que es necesario conocer qué aspectos son los que utilizan los clientes para evaluar el servicio y cuál es la percepción que tienen sobre los mismos [20], orientando la evaluación hacia el proceso más que hacia el resultado. Esta perspectiva supone admitir que la determinación de la calidad en los servicios debe estar basada fundamentalmente en las percepciones que los clientes tienen sobre este [20-22], con lo que se introduce el concepto de calidad percibida de los servicios, que ha sido la forma de conceptualizar la calidad predominante en este ámbito.

Los conceptos de calidad percibida y satisfacción están estrechamente interrelacionados, hasta el punto que algunos autores sugieren que ambos términos deben ser considerados sinónimos [23-24]. Otros autores, en tanto, como Morales y Hernández [25], indican que aún no está claro si la satisfacción de 
los clientes produce calidad percibida o si la calidad de los servicios conduce a la satisfacción. Incluso hay autores que afirman que la calidad precede y es antecedida por la satisfacción, como MartínezTur y colaboradores [26], Zeithaml y Parasuraman [27] y Oliver [28].

Específicamente, Parasuraman, Zeithaml y Berry [629-30] definen la calidad de servicio percibida como el juicio global del cliente acerca de la excelencia o superioridad de este, que resulta de la comparación entre las expectativas de los consumidores (lo que ellos creen que las empresas de servicios deben ofrecer) y sus percepciones sobre los resultados del servicio ofrecido.

Indican que a la hora de evaluar la calidad de servicio y en ausencia de criterios de carácter objetivo es necesario realizar un diagnóstico de este, a partir del análisis de las percepciones de los clientes o usuarios con relación al servicio recibido [31].

Radomir, Plaias y Nistor [32], a propósito de la conceptualización acerca de la calidad del servicio, subrayan los aspectos principales que necesitan ser tomados en cuenta cuando se intenta explicar el concepto de calidad en estos, siendo ellos los relacionados con las características que diferencian los productos de los servicios, coincidiendo con Parasuraman y colaboradores [6].

La percepción del usuario o cliente hace referencia a como este estima que la organización cumple con la entrega del servicio de acuerdo con la manera como él valora lo que recibe. Las expectativas del cliente definen lo que se espera sea el servicio que entrega la organización y se forman en base a sus experiencias pasadas, sus necesidades conscientes, comunicación de boca a boca e información externa.
A partir de aquí puede surgir una retroalimentación hacia el sistema cuando el cliente emite un juicio [33].

Es fundamental para lograr una opinión de excelente calidad de servicio, satisfacer o exceder los deseos del cliente, los cuales esperan que este tipo de organizaciones tengan una buena apariencia, sean confiables, responsables, tranquilizadoras y empáticas [34]. Por consiguiente, para que una organización logre mejorar su calidad de servicio se deben seguir dos pasos principales, el primero y el más importante corresponde a la medición de la calidad (técnica y funcional) para después centrarse en los aspectos más relevantes y/o aquellos en los que el nivel de desempeño sea menor [35].

En este sentido, en el año 1988 Parasuraman, Zeithaml y Berry [6-29-30] proponen un instrumento de medida (denominado SERVQUAL por las palabras en inglés Service Quality) para evaluar la calidad del servicio a partir de las diferencias o brechas (gap) entre las expectativas y las percepciones, caracterizadas en 5 criterios clave o dimensiones (ver Figura 1):

1. Confiabilidad: Capacidad para llevar a cabo el servicio prometido de manera confiable y precisa.

2. Capacidad de respuesta/Receptividad: Disposición de atender a las necesidades de la persona usuaria y de proporcionar un servicio pronto y oportuno.

3. Seguridad/Competencia: Conocimiento por parte de los/as servidores/as y su habilidad de inspirar confianza sobre lo que están realizando.

4. Empatía: La capacidad de percibir y comprender los requerimientos, mediante la identificación de los mismos y la atención individualizada a la persona usuaria.

Fuente: Zeithaml, Berry y Parasuraman [6].

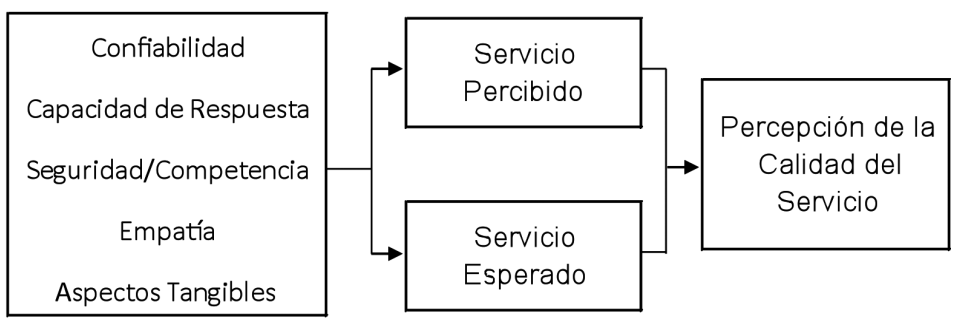

Figura 1. Dimensiones del modelo SERVQUAL. 
5. Aspectos tangibles: Apariencia y condiciones de las instalaciones físicas, equipos y apariencia del personal.

Entre los métodos existentes, la escala SERVQUAL ha sido ampliamente aceptada y utilizada en numerosos contextos, demostrando así que puede ser una herramienta eficaz y adecuada para medir la calidad del servicio en las distintas industrias [36]. Este enfoque considera un análisis de encuesta basado en la evaluación de la calidad del servicio percibida y esperada por parte de los clientes [37]. Esta escala se basa en cuestionarios aplicados de manera directa a los usuarios, incorporando aspectos cuantitativos y cualitativos agrupados en cinco dimensiones, lo que da un total de 22 declaraciones que intentan recolectar las expectativas de un excelente servicio y las percepciones sobre las mismas dimensiones de los servicios recibidos.

Cabe mencionar que las dimensiones de la escala SERVQUAL fueron creadas de manera genérica para cualquier tipo de organización, y además sólo recogen la percepción del cliente en un momento específico del tiempo [38]. Como respuesta a esto, en la literatura se encuentran autores que basados en el modelo original SERVQUAL, innovaron al adaptar el cuestionario a sectores económicos específicos. En este sentido, Babakus y Mangold [39] redujeron el número de preguntas del SERVQUAL a 15 y modificaron la escala de Likert a solo 5 puntos con el fin de reducir el nivel de frustración de los encuestados [40]. Por su parte, Cronin y Taylor [41] adaptan el cuestionario manteniendo las dimensiones originales y los 22 ítems de valoración, mientras que Richard y Allaway [42] proponen incluir variables que midan la calidad técnica (resultados) basado en el esquema propuesto de Oliver [28]. Al mismo tiempo, Izogo y Ogba [43] exponen que, si bien la escala SERVQUAL es una buena base para medir la calidad del servicio percibida, se deben realizar modificaciones para ajustar las dimensiones de acuerdo con el contexto donde se hará el estudio.

En la actualidad, las empresas e instituciones hacen cada vez un mayor uso del internet como plataforma para vender productos o entregar servicios, y a pesar de muchas veces no existir una transacción o acción persona a persona, los clientes aún muestran preocupación por recibir un servicio de calidad, especialmente cuando se trata de la resolución de problemas [44]. Lo anterior nos indica que, si bien los medios o plataformas utilizadas varían en el tiempo, seguirá siendo relevante para estas organizaciones el estudiar cómo perciben sus clientes la calidad del servicio entregado.

Debido a lo expuesto previamente, diversos estudios exploran la relación entre la calidad y la satisfacción de los usuarios mediante el uso de la escala SERVQUAL. De esta forma, Basfirinci y Mitra [45] midieron la calidad del servicio de aerolíneas en Estados Unidos y Turquía, integrando la escala SERVQUAL con el modelo Kano, con lo cual mostraron que las diferencias culturales pueden definir las expectativas y percepciones de los clientes.

Por otro lado, en el ámbito de los servicios públicos, por ejemplo, Ladhari y Rigaux-Bricmont [46] conducen un estudio en hospitales públicos de Canadá mediante la utilización del modelo SERVQUAL, comprobando que la calidad percibida por los pacientes tiene un efecto directo en la satisfacción de estos. Chatzoglou y colaboradores [47] utilizan una versión ligeramente modificada de SERVQUAL para medir la calidad de los Centros de Atención al Ciudadano en Grecia. Finalmente, Liu y colaboradores [48] también utilizan este modelo para analizar la calidad de la Compañía de Certificación e Inspección de China (CCIC).

\section{METODOLOGÍA}

El tipo de investigación del presente estudio es de carácter exploratorio, ya que es un tema poco estudiado y del que no se han encontrado investigaciones previas en el mismo ámbito y contexto, al mismo tiempo es descriptivo debido a que busca especificar las propiedades y características de la calidad percibida en el servicio judicial de Puerto Montt.

De igual manera, el estudio es de tipo transversal, dado que se tomaron los datos en dos periodos específicos de tiempo (diciembre de 2013 y junio de 2018), con la intención de evaluar la evolución de la percepción por parte de los usuarios [49].

\section{Diseño del instrumento}

Se evaluó la calidad percibida de los usuarios por medio de la técnica SERVQUAL a través de un cuestionario, el que recogió 16 indicadores que 
representan las cinco dimensiones señaladas por Parasuraman, Zeithaml y Berry [30], medidos en una escala Likert de 5 puntos que va desde " $5=$ Muy de acuerdo hasta 1= Muy en desacuerdo". Si bien el modelo plantea 22 indicadores fue precisa la adaptación de los ítems, añadiendo unos y eliminando otros, para reflejar mejor las características específicas del servicio.

El cuestionario incluyó perfil de los encuestados: sexo, edad, residencia, nivel educacional y motivo por el cual se encontraba como usuario en el juzgado de garantía al momento de aplicación del mismo.

Previo a la aplicación del instrumento se realizó una encuesta piloto donde participaron 10 usuarios del juzgado de garantía, con el objeto de validar su contenido y mejorar la redacción de las preguntas. En la Tabla 1 se puede observar el cuestionario final, el cual evalúa la importancia de 16 componentes de calidad de servicio, que representan las dimensiones del SERVQUAL.

Por último, la consistencia interna del instrumento se verificó por medio del examen Alfa de Cronbach que se basa en la correlación media entre los puntos, el que arrojó un valor de 0,873 para las expectativas y 0,893 para las percepciones, lo que indica una adecuada fiabilidad del instrumento.

\section{Participantes y procedimientos de recolección}

El cuestionario se aplicó a las personas mayores de 18 años, que se encontraban como usuarios dentro del juzgado de garantía de Puerto Montt entre el 10 y 18 de diciembre de 2013 y entre el 20 y el 26 de junio de 2018. Se empleó, en ambos casos muestreo por conveniencia debido a que se dependía mayormente de la disposición de estos a realizar la encuesta, obteniéndose un total de 107 respuestas.

Finalmente, el método de recogida de la información fue la encuesta presencial, donde los entrevistadores primeramente se presentaban a los usuarios y les ofrecían una breve descripción de la intención del estudio, para luego solicitar su colaboración para rellenar el cuestionario con sus respectivas opiniones.

\section{RESULTADOS}

A continuación, se presentan los resultados de mayor relevancia del estudio, estos fueron analizados sobre la base de la media y se identificó la diferencia (gap) entre la expectativa y la percepción del usuario.

Tal como puede observarse en la Tabla 2, más del $80 \%$ de los encuestados tiene su residencia en la zona urbana, más del $50 \%$ son varones, más del $70 \%$ tiene educación media, técnica profesional o superior y cerca del $60 \%$ pertenecen al grupo etario

Tabla 1. Dimensiones e indicadores.

\begin{tabular}{|c|c|}
\hline Dimensión & Indicadores \\
\hline Confiabilidad & $\begin{array}{l}\text { C1: Confiabilidad del juzgado de garantía. } \\
\text { C2: Cumplimiento de los horarios establecidos. } \\
\text { C3: Información clara y precisa. }\end{array}$ \\
\hline Capacidad de Respuesta & $\begin{array}{l}\text { CR1: Conclusión de trámites en tiempo razonable. } \\
\text { CR2: Atención rápida y expedita. } \\
\text { CR3: Disposición de los funcionarios para resolver dudas e inquietudes. }\end{array}$ \\
\hline Seguridad/Competencia & $\begin{array}{l}\text { S1: Posesión de las competencias por parte de los funcionarios para ofrecer un } \\
\text { servicio adecuado. } \\
\text { S2: Competencia de fiscales en la protección de víctimas y delitos. } \\
\text { S3: Competencia de los defensores públicos en la asistencia y defensa de los } \\
\text { imputados. } \\
\text { S4: Competencia de jueces para impartir justicia. }\end{array}$ \\
\hline Empatía & $\begin{array}{l}\text { E1: Interés de los funcionarios por las inquietudes planteadas por los usuarios. } \\
\text { E2: Atención y cortesía de los funcionarios. } \\
\text { E3: Respeto de parte de los funcionarios. }\end{array}$ \\
\hline Aspectos tangibles & $\begin{array}{l}\text { AT1: Estado de la infraestructura del juzgado. } \\
\text { AT2: Estado de equipos e instalaciones. } \\
\text { AT3: Nivel de seguridad al interior del juzgado. }\end{array}$ \\
\hline
\end{tabular}

Fuente: Diseño propio, adaptado de Parasuraman, Zeithaml y Berry [6]. 
Tabla 2. Perfil de los encuestados.

\begin{tabular}{|c|c|c|c|c|}
\hline \multicolumn{5}{|c|}{$2013-2018$} \\
\hline \multicolumn{5}{|c|}{ Residencia } \\
\hline \multicolumn{2}{|c|}{ Urbana } & \multicolumn{3}{|c|}{ Rural } \\
\hline \multicolumn{2}{|c|}{$81,3 \%-83,2 \%$} & \multicolumn{3}{|c|}{$18,7 \%-16,8 \%$} \\
\hline \multicolumn{5}{|c|}{ Sexo } \\
\hline \multicolumn{2}{|c|}{ Hombre } & \multicolumn{3}{|c|}{ Mujer } \\
\hline \multicolumn{2}{|c|}{$54 \%-57 \%$} & \multicolumn{3}{|c|}{$46 \%-43 \%$} \\
\hline \multicolumn{5}{|c|}{ Nivel Educacional } \\
\hline Educación Universitaria & Técnico Profesional & Educación Media & Educación Básica & Sin Escolaridad \\
\hline $17,8 \%-20,6 \%$ & $7,5 \%-8,4 \%$ & $52,3 \%-47,7 \%$ & $18,7 \%-20,6 \%$ & $3,7 \%-2,8 \%$ \\
\hline \multicolumn{5}{|c|}{ Edad } \\
\hline 18 a 29 años & 30 a 39 años & 40 a 49 años & 50 a 59 años & 60 años o más \\
\hline $34,6 \%-21,5 \%$ & $23,4 \%-43,0 \%$ & $25,2 \%-16,8 \%$ & $10,3 \%-15,0 \%$ & $6,5 \%-3,75 \%$ \\
\hline \multicolumn{5}{|c|}{ Cuál es el motivo por el que se encuentra en el Juzgado de Garantía } \\
\hline Víctima & Imputado & Acompaña a familiar & Acompaña a amigo & Otro \\
\hline $28 \%-24,3 \%$ & $24,3 \%-18,7 \%$ & $18,7 \%-30,8 \%$ & $3,7 \%-15 \%$ & $25,2 \%-11,2 \%$ \\
\hline
\end{tabular}

Fuente: Diseño propio, basado en encuesta de percepción.

que tiene entre 18 y 39 años. En cuanto a la razón por la cual se encontraban en el juzgado de garantía al momento de aplicar el cuestionario, sobre el $20 \%$ se identificó como "víctima" o como "imputado".

Los resultados de las expectativas promedio de la calidad de servicio del juzgado de garantía, se analizaron de forma global, por dimensión y por el motivo por el cual se encontraba en el lugar. La nota global para la muestra de encuestados, en cuanto a expectativas fue de 4,1 en 2013 y de 4,0 en 2018, lo que señala que las expectativas de los usuarios con relación a la calidad del servicio son elevadas.
Sin embargo, la percepción real de los encuestados con respecto a la calidad del servicio fue de 3,8 en 2013 y de 3,6 en 2018, lo que no solo significa que la percepción no supera las expectativas, sino que esta ha disminuido en el tiempo.

En la Tabla 3, se muestran los resultados obtenidos a nivel de expectativas y percepciones para cada una de las dimensiones. En efecto, a nivel de expectativas, los valores más altos se encuentran en las dimensiones Confiabilidad, Seguridad y Aspectos Tangibles. Cabe hacer notar que las expectativas promedio de los usuarios disminuyeron en la medición realizada el

Tabla 3. Expectativas y Percepciones por Dimensiones (2013-2018).

\begin{tabular}{|l|c|c|c|c|}
\hline \multirow{2}{*}{\multicolumn{1}{|c|}{ Dimensión }} & \multicolumn{2}{c|}{ Expectativas } & \multicolumn{2}{c|}{ Percepciones } \\
\cline { 2 - 5 } & $\mathbf{2 0 1 3}$ & $\mathbf{2 0 1 8}$ & $\mathbf{2 0 1 3}$ & $\mathbf{2 0 1 8}$ \\
\hline Confiabilidad & 4,2 & 4,0 & 3,8 & 3,6 \\
\hline Capacidad de Respuesta & 3,8 & 3,6 & 3,6 & 3,5 \\
\hline Seguridad & 4,2 & 4,0 & 3,7 & 3,4 \\
\hline Empatía & 4,1 & 4,0 & 4,1 & 3,7 \\
\hline Aspectos Tangibles & 4,2 & 4,3 & 4,1 & 3,8 \\
\hline
\end{tabular}

Fuente: Diseño propio, basado en encuesta realizada. 
año 2018 para todas las dimensiones con excepción de los Aspectos Tangibles. Asimismo, la dimensión con menor expectativa; Capacidad de Respuesta se reduce aún más en la segunda medición.

Por otro lado, con respecto a la calidad percibida promedio, si se analizan las dimensiones, se puede apreciar que el valor más bajo para el año 2013, correspondió a la Dimensión Capacidad de Respuesta (rapidez y disposición de ayuda al usuario), seguida de Seguridad (profesionalidad, cortesía y credibilidad que generan los empleados) y Confiabilidad (cumplimiento de lo prometido). Al analizar los datos 2018, la dimensión con más baja percepción fue Seguridad/Competencia.

Luego, si se examinan las expectativas promedio por indicador, en la Tabla 4, se observa que las expectativas más elevadas para el año 2013 se encontraban en los indicadores AT1 (estado de la infraestructura) con un promedio de 4,5 y S1 (posesión de las competencias por parte de los funcionarios para ofrecer un servicio adecuado) con un 4,4. En la medición 2018 las expectativas promedio por indicador disminuyen, a excepción de AT2 y AT3, las cuales corresponden al estado de equipos e instalaciones y al nivel de seguridad al interior del juzgado, las cuales aumentan.
Mientras que, al analizar las percepciones, el valor más bajo para el año 2013 pertenece al indicador CR1 (conclusión de trámites en tiempo razonable). Mientras que los niveles más altos de percepción se asocian a los indicadores E1 (interés de los funcionarios por las inquietudes planteadas por los usuarios), E3 (respeto por parte de los funcionarios) y AT3 (seguridad al interior del juzgado) con un valor de 4,2. Asimismo, hacia el año 2018 la percepción de los usuarios ha disminuido mayormente, a excepción de los indicadores C3 (información clara y precisa) y CR1, los cuales mejoran levemente.

También se calcularon los gaps o brechas absolutas por dimensión e indicadores entre las expectativas y la calidad percibida. Como se observa en la Figura 2, para el año 2013 la mayor brecha se presenta en las dimensiones de Seguridad y Confiabilidad con un valor de 0,4 , es decir, los usuarios esperan mayores competencias de los funcionarios, fiscales, defensores y jueces. Asimismo, estos perciben que el Juzgado no cumple sus expectativas con respecto al servicio de justicia requerido. De acuerdo a la encuesta aplicada en 2018, los Gaps en la mayor parte de las Dimensiones se incrementan con excepción de Capacidad de Respuesta y Confiabilidad, es decir los usuarios perciben que el Juzgado no cumple sus expectativas de servicio.

Tabla 4. Expectativas y percepciones por indicador (2013-2018).

\begin{tabular}{|l|c|c|c|c|c|}
\hline \multirow{2}{*}{} & \multicolumn{2}{c|}{ Expectativas } & \multicolumn{2}{c|}{ Percepciones } \\
\cline { 3 - 6 } & & $\mathbf{2 0 1 3}$ & $\mathbf{2 0 1 8}$ & $\mathbf{2 0 1 3}$ & $\mathbf{2 0 1 8}$ \\
\hline \multirow{3}{*}{ Confiabilidad } & C1 & 4,3 & 4,2 & 3,6 & 3,4 \\
\cline { 2 - 6 } & C2 & 4,0 & 3,8 & 4,0 & 3,4 \\
\cline { 2 - 6 } & C3 & 4,3 & 4,0 & 3,8 & 4,0 \\
\hline \multirow{4}{*}{ Capacidad de Respuesta } & CR1 & 3,5 & 3,2 & 3,0 & 3,1 \\
\cline { 2 - 6 } & CR2 & 4,0 & 3,8 & 3,9 & 3,7 \\
\cline { 2 - 6 } & CR3 & 4,0 & 3,8 & 3,8 & 3,7 \\
\hline \multirow{4}{*}{ Seguridad } & S1 & 4,4 & 4,2 & 3,9 & 3,4 \\
\cline { 2 - 6 } & S2 & 4,0 & 3,8 & 3,7 & 3,4 \\
\cline { 2 - 6 } & S3 & 4,0 & 3,8 & 3,5 & 3,4 \\
\cline { 2 - 6 } & S4 & 4,2 & 4,0 & 3,8 & 3,4 \\
\hline \multirow{3}{*}{ Empatía } & E1 & 4,0 & 4,0 & 4,2 & 3,8 \\
\cline { 2 - 6 } & E2 & 4,3 & 4,2 & 3,8 & 3,5 \\
\cline { 2 - 6 } & E3 & 4,0 & 3,8 & 4,2 & 3,7 \\
\hline \multirow{3}{*}{ Aspectos Tangibles } & AT1 & 4,5 & 4,2 & 4,1 & 3,9 \\
\cline { 2 - 6 } & AT2 & 4,2 & 4,4 & 4,0 & 3,8 \\
\cline { 2 - 6 } & AT3 & 4,0 & 4,2 & 4,2 & 3,8 \\
\hline
\end{tabular}

Fuente: Diseño propio, basado en encuesta realizada. 
Fuente: Diseño propio, basado en encuesta realizada.

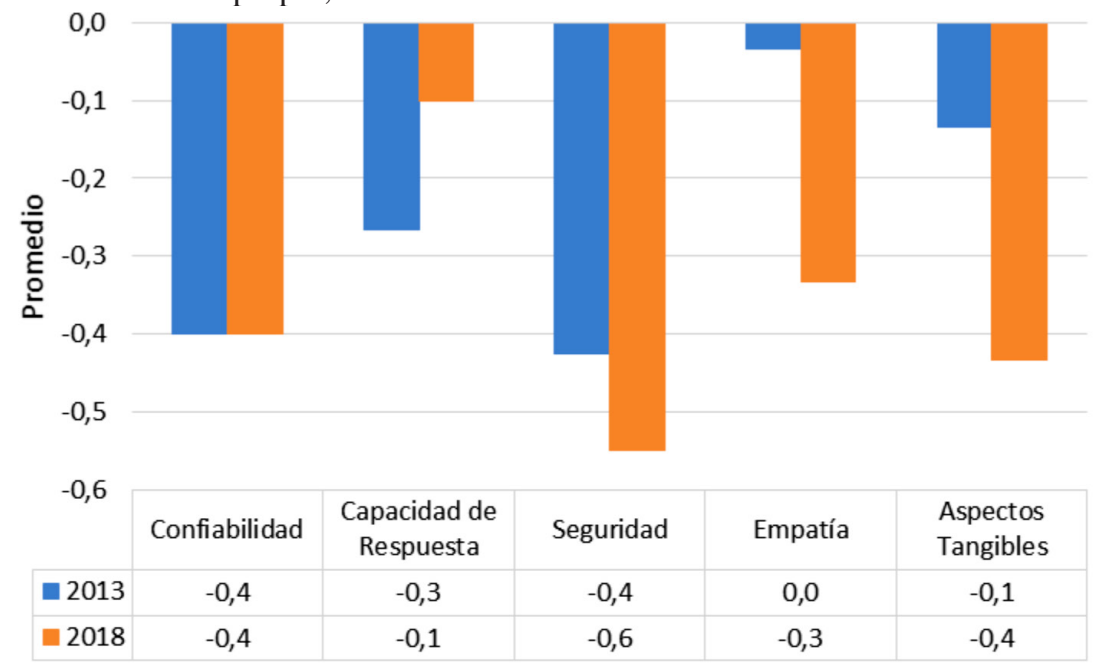

Figura 2. Gaps por dimensión (2013-2018).

Un mayor nivel de detalle se presenta en la Figura 3, en la cual se visualiza claramente que la mayor brecha negativa entre calidad esperada y calidad percibida, para el año 2013, se encuentra en el indicador C1 (confiabilidad en el juzgado de garantía), la cual es superior a lo que se espera del tribunal. La mayor brecha positiva o gap se alcanza en los indicadores E1 y E3 que dan cuenta del interés y respeto que muestran los funcionarios del juzgado. El indicador AT3 también presenta una brecha positiva, es decir, se percibe una mayor seguridad al interior del juzgado que aquella que se espera al ingresar a él. Por otro lado, para el año 2018, no existen brechas positivas, solo el indicador CR3 (disposición de los funcionarios para resolver dudas e inquietudes) no presenta brecha.

De manera adicional al análisis anterior se calcularon las brechas o gaps para los indicadores separando el tipo de usuarios en víctimas e imputados, como se puede observar en la Figura 4 y 5.

En la Figura 4 se puede observar que para el año 2013 la calificación global promedio para las expectativas que tenían los usuarios "víctimas" fue de 4,14 y para la calidad percibida fue de 3,87 , ambos

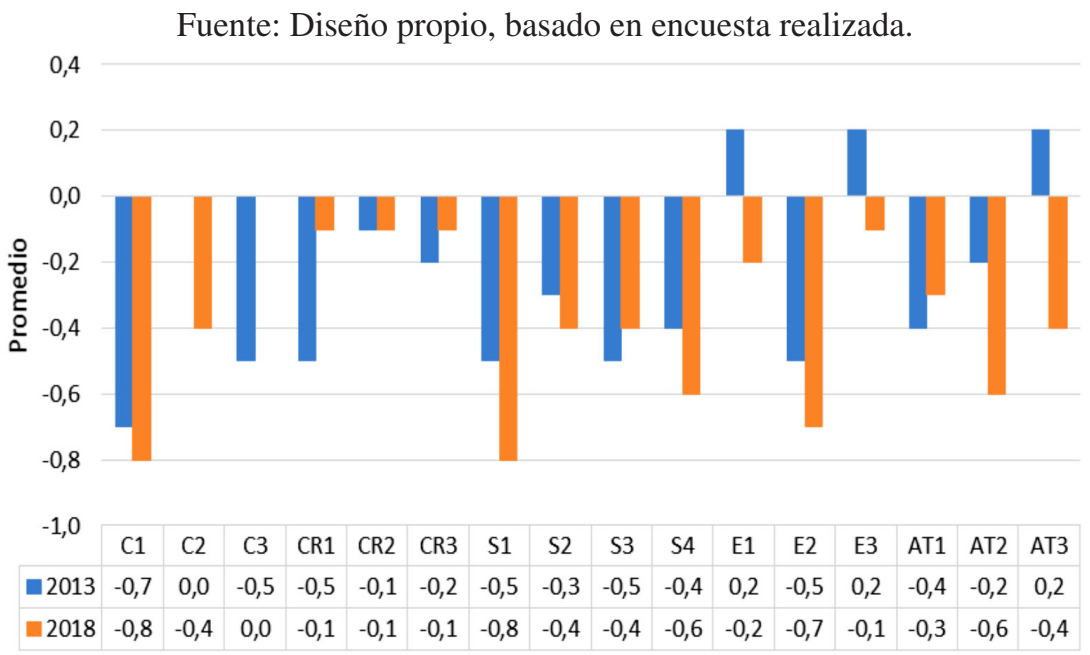

Figura 3. Gaps por indicador (2013-2018). 


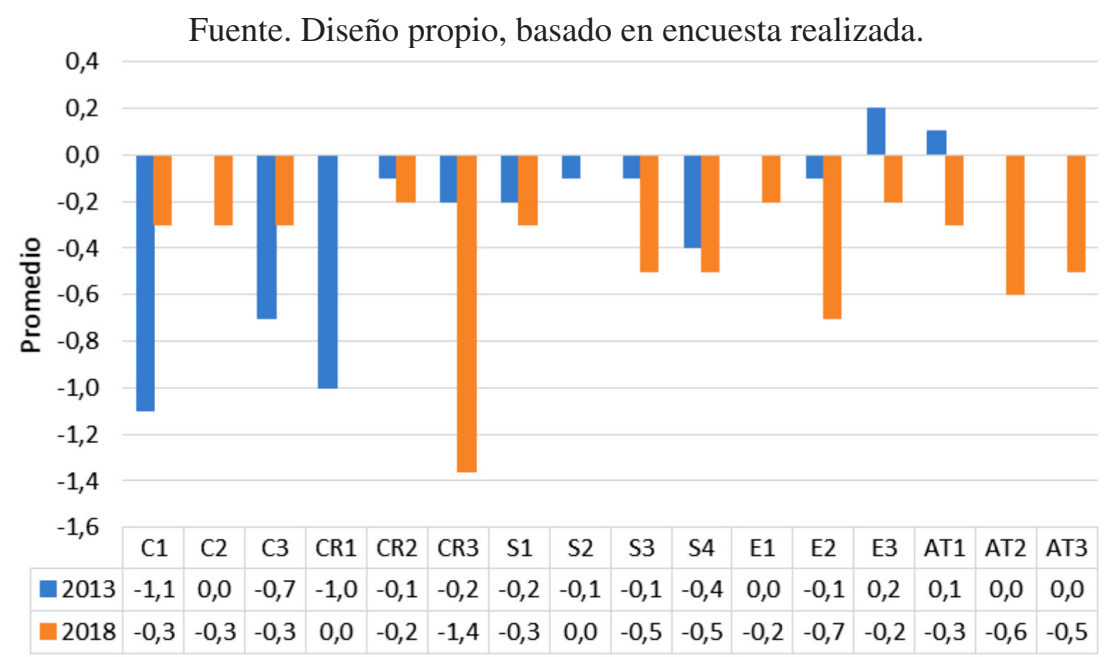

Figura 4. Gaps por indicador (Víctimas 2013-2018).

valores levemente superiores al de la totalidad de usuarios. Los gaps más elevados se presentan en los indicadores $\mathrm{C} 1$ (confiabilidad en el juzgado de garantía) y CR1 (conclusión de trámites en tiempo razonable). En tanto para 2018, los mayores gaps se encuentran en CR3 (disposición de los funcionarios para resolver dudas e inquietudes) y E2 (atención y cortesía de los funcionarios).

En el caso de los imputados, y tal como se muestra en la Figura 5, el mayor gap se presenta, para el 2013, en el indicador E2 el cual da cuenta del interés de los funcionarios por las inquietudes planteadas por los usuarios, la atención, cortesía y respeto de parte de estos. Asimismo, el mayor gap positivo que se presenta es para CR1, lo que significa que la percepción de los imputados sobre la rapidez en la que se concluyen los trámites supera sus expectativas.

En la encuesta aplicada en 2018 los mayores gaps se presentan en los indicadores S3: Competencia de los defensores públicos en la asistencia y defensa de los imputados $(-0,8)$, CR2 y AT2 $(-0,7)$. Claramente la calidad percibida de los imputados en la mayor parte de los indicadores es menor que la de las víctimas, no obstante, los imputados mantienen una mejor percepción respecto del trabajo de los Jueces al momento de aplicar sentencia, en tanto que las

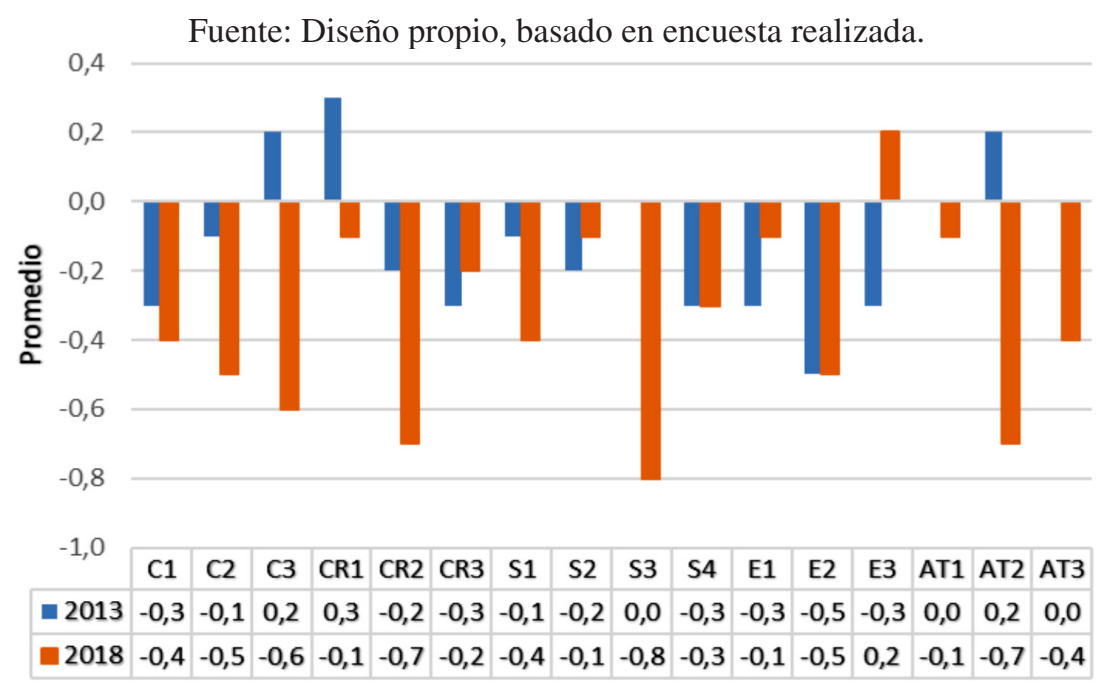

Figura 5. Gaps por indicador (Imputados 2013-2018). 
víctimas lo evalúan de peor forma (S4: Competencia de jueces para impartir justicia).

\section{DISCUSIÓN DE RESULTADOS}

En su mayoría, las expectativas del usuario son más altas que las percepciones, por lo que se desprende que el juzgado de garantía de Puerto Montt no está alcanzando los niveles de calidad requeridos por estos. Esto mismo ha llevado a que el puntaje asociado a las expectativas vaya disminuyendo a lo largo de los años.

Para el caso del análisis por dimensiones llevado a cabo, se puede decir, que los usuarios esperan en mayor medida, que el juzgado cumpla con su "deber" de impartir justicia, que los funcionarios, fiscales, defensores y jueces sean competentes y que las instalaciones ofrezcan condiciones adecuadas y de seguridad para su funcionamiento. Esto último puede ser explicado por el hecho de que el Juzgado de Garantía de Puerto Montt funciona en un moderno edificio inaugurado en 2010, ubicado en un lugar de fácil acceso, y ampliamente reconocible para los habitantes de la ciudad. En cuanto al análisis de los indicadores, se entiende que los usuarios esperen un mayor nivel de seguridad e instalaciones modernas debido a lo mencionado anteriormente.

Por otro lado, se evidencia que las cinco dimensiones han disminuido su nivel de apreciación en el año 2018 en comparación al 2013, lo que implica que los usuarios perciben un desgaste de servicio a nivel global. Resaltando la seguridad como la dimensión con el menor puntaje, lo que significa que existe un deterioro evidente en la percepción de las capacidades de los funcionarios, defensores y fiscales para ofrecer un servicio adecuado a víctimas e imputados y en impartir justicia por parte de los jueces. A nivel de indicadores la mayoría sufrió una disminución de los niveles de percepción de los usuarios, sin embargo, estos consideran que los funcionarios mejoraron su desempeño al lograr entregar la información de forma más clara y precisa y desempeñar sus labores en un tiempo razonable.

\section{CONCLUSIONES}

El poder judicial, así como otras entidades públicas en Chile, constantemente se encuentra bajo el foco de atención debido a los constantes problemas relacionados con la administración, burocracia y atención a los usuarios. Es por esto que la evaluación de la calidad de los servicios debe tener en cuenta la perspectiva de quienes los utilizan. En este proceso de evaluación entran en juego sus expectativas, de manera que los usuarios comparan el servicio prestado por la administración con lo que espera de él antes de recibirlo. Estas expectativas se forman a partir de las experiencias anteriores, propias o ajenas en relación con dicho servicio.

Como se planteó anteriormente, la calidad para ser mejorada, primero debe ser medida para luego mejorar aquellos aspectos en los que la organización desee enfocarse, por esta razón el modelo SERVQUAL empleado en la presente investigación se alza como una buena alternativa para identificar los atributos de un servicio en torno a los cuales se configuran las expectativas de los usuarios.

Los niveles de calidad del servicio en el juzgado de garantía, medidos a través de la puntuación de la escala de SERVQUAL, se muestran desfavorables o negativos en su globalidad al no ser superadas las expectativas del usuario por sus percepciones, lo que significa que el servicio que está entregando este juzgado no es considerado de calidad por parte de la mayoría de los usuarios.

La valiosa información de la evaluación de la calidad del servicio, puede ser convenientemente usada por la organización, en este caso el juzgado de garantía de Puerto Montt, para mejorar los atributos de mayor relevancia para los usuarios encuestados, como la confiabilidad y la capacidad de respuesta, implementando los planes de acción que se consideren pertinentes para el logro de mejores niveles de calidad.

Si bien el modelo SERVQUAL es muy utilizado para la medición de los niveles de calidad en los servicios, está poco explorado en el ámbito judicial, especialmente en el contexto chileno, por lo que este estudio pretende pavimentar el camino para futuras investigaciones dentro de este ámbito, ya sea ampliando el rango geográfico y/o sectorial.

\section{REFERENCIAS}

[1] J. Westphal, R. Gulati, and S. Shortell. "Customization or conformity: an institutional 
and network perspective on the content and consequences of TQM adoption". Administrative Science Quarterly. Vol. 42, pp. 366-394. 1997. DOI: 10.2307/2393924.

[2] A. Meesala and J. Paul. "Service quality, consumer satisfaction and loyalty in hospitals: Thinking for the future". Journal of Retailing and Consumer Services. Vol. 40, pp. 261-269. 2018. DOI: 10.1016/j.jretconser.2016.10.011.

[3] Real Academia Española. "Calidad". En Diccionario de la lengua española (23.a ed.). Recuperado de: http://dle.rae. es/?id=6nVpk8P|6nXVL1Z.

[4] L. Ribeiro. "Gestão da Qualidade: Conceitos e Técnicas”. Editorial Atlas. 3ra Ed. Vol.1. São Paulo, Brasil. ISBN: 978-85-970-0391-8. 2010.

[5] J. Toharia y J. García de la Cruz. "La Justicia ante el espejo: 25 años de estudios de opinión del CGPJ". Editores Consejo General del Poder Judicial. España. ISBN: 84-9651825-6. 2005.

[6] A. Parasuraman, V. Zeithaml, and L. Berry. "SERVQUAL: A multiple-item scale for measuring consumer perceptions of service quality". Journal of Retailing. Vol. 64, Issue 1, pp. 12-40. 1988.

[7] S. Akter, J. D'Ambra and P. Ray. "Service quality of Health platforms: development and validation of a hierarchical model using PLS". Electronic Markets. Vol. 20, Issue 3-4, pp. 209227. 2010. DOI: 10.1007/s12525- 010-0043-x.

[8] A. Huang, K. Chen, D. Yen and T. Tran. "A study of factors that contribute to online review helpfulness". Computers in Human Behavior. Vol. 48, pp. 17-27. 2015. DOI: 10.1016/ j.chb.2015.01.010.

[9] F. Murillo-Torrecilla y R. Hernández-Castilla. "La red iberoamericana de investigación sobre cambio y eficacia escolar. Lecciones aprendidas del funcionamiento de la red". Revista Iberoamericana sobre Calidad, Eficacia y Cambio en Educación. Vol. 10 No 3, pp. 144-151. 2012. ISSN: 1696-4713.

[10] E. Cano, X. Chavarria, X. Gimeno y J. Teixido. "La calidad de la educación: los peligros de un discurso seductor". En VIII jornadas sobre Dirección Escolar. Cataluña. España. 24 y 25 de mayo 2002.

[11] D. Gallego y C. Alonso. "Estilos de aprender en el siglo XXI". Revista Estilos de Aprendizaje. Vol. 2 № 2, pp. 2-13. 2008.
[12] O. Colmenare y J. Saavedra. “Aproximación teórica de los modelos conceptuales de la calidad del servicio". Técnica Administrativa. Vol. $6 \mathrm{~N}^{\circ}$ 32. 2007. ISSN-e 1666-1680.

[13] J. Vera, A. Trujillo. "El efecto de la calidad del servicio en la satisfacción del derechohabiente en instituciones públicas de salud en México". Contaduría y administración. Vol. $63 \mathrm{~N}^{\circ}$ 2, pp. 1-22. 2018. DOI: 10.1016/j. cya.2016.07.003.

[14] L. Pedraja-Rejas y E. Rodríguez-Ponce. "La relevancia del proyecto estratégico como determinante de la calidad en las universidades". Idesia (Arica). Vol. 32 No 4, pp. 101-103. 2014. DOI: $10.4067 /$ S0718-34292014000400013.

[15] L. Pedraja-Rejas y E. Rodríguez-Ponce. "El aseguramiento de la calidad: Un imperativo estratégico en la educación universitaria". Ingeniare Revista Chilena de Ingeniería. Vol. 23, No 1, pp. 4-5. 2015. DOI: 10.4067/ S0718-33052015000100001.

[16] M. Lemaitre. Aseguramiento de la calidad: Una política y sus circunstancias. En A. Bernasconi (Ed.), Ediciones UC. "La educación superior de Chile: Transformación, desarrollo y crisis". 3ra ed. Vol. 1. pp. 295-344. Santiago, Chile. 2015. ISBN: 978-956-14-1492-1.

[17] E. Rebolloso, C. Salvador, B. Fernández y P. Cantón. "Análisis y ampliación del SERVQUAL en los servicios universitarios". Revista de Psicología del Trabajo y de las Organizaciones. Vol. 20, No 3, pp. 355-373. 2004. ISSN 1576-5962.

[18] M. Kumar, K. Sujit and V. Charles. "Deriving managerial implications through SERVQUAL gap elasticity in UAE banking", International Journal of Quality \& Reliability Management, Vol. 35 Issue 4, pp. 940-964. 2018. DOI: 10.1108/IJQRM-10-2016-0176.

[19] J. Fatima and M. Razzaque. "Service quality and satisfaction in the banking sector". International Journal of Quality \& Reliability Management. Vol. 31, Issue 4, pp. 367-379. 2014. DOI: 10.1108/IJQRM-02-2013-0031.

[20] C. Grönroos. "A service quality model and its marketing implementations". European Journal of Marketing. Vol. 18, Issue 4, pp. 36-44. 1984.

[21] A. Parasuraman, V. Zeithaml and L.L. Berry. “A Conceptual Model of Service Quality 
and its Implications for Future Research". Journal of Marketing. Vol. 49, Issue 4, pp. 41-50. 1985.

[22] J.B. Steenkamp. "A conceptual model of quality perception process". Journal of Business Research. Vol. 21, pp. 309-333. 1990. DOI: 10.1108/EUM0000000004784.

[23] V. Liljander. "Modeling perceived service quality using different comparison standards". Journal of Consumer Satisfaction, Dissatisfaction and Complaining Behaviour. Vol. 7, pp. 126-142. 1994.

[24] P. Dabholkar. "A contingency framework for predicting causality between customer satisfaction and service quality". En F. R. Karden y M. Sujan (eds.), Advances in consumer research, Vol. 22, pp. 101-108. 1995.

[25] V. Morales y A. Hernández. "Calidad y satisfacción en los servicios: conceptualización". Lecturas: Educación Física y Deportes. Vol. 10, Issue 73. 2004. ISSN-e 1514-3465.

[26] V. Martínez-Tur, J. M. Peiró y J. Ramos. "Calidad de servicio y satisfacción del cliente". Síntesis. Madrid, España. ISBN: 9788477388869. 2001.

[27] V.A. Zeithaml and A. Parasuraman. "Relevant knowledge series: Service Quality". Marketing Science Institute. Cambridge, USA. ISBN: 0965711439. 2004.

[28] R.L. Oliver. "An investigation of the interrelationship between consumer (dis)satisfaction and complaint reports". In M. Wallendorf and P. Anderson (Eds), Advances in Consumer Research, Vol. 14, pp. 218-222. Provo, USA. 1987.

[29] A. Parasuraman, V. A. Zeithaml, and L. L. Berry. "Research note: more on improving service quality measurement". Journal of Retailing. Vol. 69, Issue 1, pp. 140-147. 1993. DOI: 10.1016/S0022-4359(05)80007-7.

[30] A. Parasuraman, V.A. Zeithaml, and L. L. Berry. "The Behavioral Consequences of Service Quality”. Journal of Marketing. Vol. 60, Issue 2, pp. 31-46. 1996. DOI: 10.2307/1251929.

[31] R. Matsumoto. "Desarrollo del Modelo Servqual para la medición de la calidad del servicio en la empresa de publicidad Ayuda Experto". Perspectivas. No 34, pp. 181-209. 2014. ISSN: 1994-3733.

[32] L. Radomir, I. Plaias and V. Nistor. "A review of the service quality concept-past, present and perspectives". Marketing-from information to decision. Issue 5, pp. 404427. 2012.

[33] M. E. Castillo. "Escala multidimensional SERVQUAL". Recopilación. Facultad de Ciencias Empresariales. Universidad del Bío-Bío. Chile. 2005.

[34] Y. Riquelme. "Modelo de Calidad del Servicio en Centro Salud Familiar Paulina Avendaño Pereda”. Trabajo de Título. Universidad del Bío-Bío. Concepción, Chile. 2009.

[35] J. Rezaei, O. Kothadiya, L. Tavasszy and M. Kroesen. "Quality assessment of airline baggage handling systems using SERVQUAL and BWM". Tourism Management. Vol. 66, pp. 85-93. DOI: 10.1016/j.tourman.2017.11.009. 2018.

[36] M. Jebraeily, B. Rahimi, Z. Zare Fazlollahi and H. Lotfnezhad Afshar. "Using SERVQUAL Model to Assess Hospital Information System Service Quality". Hormozgan Medical Journal. Vol. 23, Issue 1, pp. 1-6. 2019. DOI: 10.5812/hmj.86977.

[37] P. Demir, M. Gul and A. Fuat Guneri. "Evaluating occupational health and safety service quality by SERVQUAL: a field survey study". Total Quality Management \& Business Excellence. 2018. DOI: 10.1080/14783363.2018.1433029.

[38] A. L. Martins, J. C. de Carvalho, T. Ramos, and J. Fael. "Assessing Obstetrics Perceived Service Quality at a Public Hospital." Procedia-Social and Behavioral Sciences, Vol. 181, pp. 414-422. 2015. DOI: 10.1016/j. sbspro.2015.04.904.

[39] E. Babakus and W.G. Mangold. "Adapting the SERVQUAL scale to hospital services: an empirical investigation". Health services research. Vol. 26, Issue 6, pp. 767-786. 1992.

[40] J.C. Vergara, V. Quesada y I. Blanco. "Factores clave para la valoración de la calidad del servicio y satisfacción del cliente: modelos causales, desarrollo y evolución". Revista Virtual Universidad Católica del Norte. Vol. 35, pp. 380-400. 2012. ISSN: 0124-5821.

[41] J.J. Cronin and S. A. Taylor. "Measuring Quality: A Reexamination and Extension". Journal of Marketing. Vol. 56, Issue 3, pp. 55-68. 1992. DOI: $10.1177 / 002224299205600304$. 
[42] M. D. Richard and A. W. Allaway. "Service quality attributes and choice behavior". Journal of Services Marketing. Vol. 7 , Issue 1, pp. 59-68. 1993. DOI: 10.1108/ 08876049310026105.

[43] E.E. Izogo and I. Ogba. "Service quality, customer satisfaction and loyalty in automobile repair services sector". International Journal of Quality \& Reliability Management. Vol. 32, Issue 3, pp. 250-269. 2015. DOI: 10.1108/ IJQRM-05-2013-0075.

[44] J.E. Collier and C.C. Bienstock. "A conceptual framework for measuring e-service quality". En H. Spotts (Ed.) Creating and Delivering Value in Marketing, pp. 158-162. Springer International Publishing. 2015. DOI: 10.1007/978-3-319-11848-2_52.

[45] C. Basfirinci and A. Mitra. "A cross cultural investigation of airlines service quality through integration of Servqual and the Kano model". Journal of Air Transport Management, Vol. 42, pp. 239-248. 2015. DOI: 10.1016/j.jairtraman.2014.11.005.
[46] R. Ladhari and B. Rigaux-Bricmont. "Determinants of patient satisfaction with public hospital services". Health marketing quarterly. Vol. 30, Issue 4, pp. 299-318. 2014. DOI: $10.1080 / 07359683.2013 .844015$.

[47] P. Chatzoglou, D. Chatzoudes, E. Vraimaki, and A. Diamantidis. "Service quality in the public sector: the case of the Citizen's Service Centers (CSCs) of Greece". International Journal of Productivity and Performance Management. Vol. 62, Issue 6, pp. 583-605. 2013. DOI: 10.1108/ IJPPM-12-2012-0140.

[48] R. Liu, L. Cui, G. Zeng, H. Wu, C. Wang, S. Yan and B. Yan. "Applying the fuzzy SERVQUAL method to measure the service quality in certification \& inspection industry". Applied Soft Computing. Vol. 26, pp. 508512. 2015. DOI: 10.1016/j.asoc.2014.10.014.

[49] R. Hernández, C. Fernández y P. Baptista. "Metodología de la Investigación". Editorial McGraw Hill. 6ta ed. Distrito Federal, México. ISBN: 978-1-4562-2396-0. 